\title{
EVALUACIÓN DEL POTENCIAL PRODUCTIVO DEL SORGO NEGRO FORRAJERO (Sorghum almum) ${ }^{1}$
}

\author{
Eugenio Araya ${ }^{2}$, Carlos Jiménez ${ }^{3}$, Henry Soto ${ }^{3}$, Anaité Quan $^{2}$
}

\begin{abstract}
RESUMEN
Evaluación del potencial productivo del sorgo negro forrajero (Sorghum almum). Por medio de cortes cada 63 días se obtuvo información sobre la productividad y composición nutricional del sorgo negro forrajero. El primer corte se realizó en los meses de octubre-noviembre de 1991, el segundo corte se realizó en los meses de diciembre 1991 y enero de 1992, el tercer corte se realizó en los meses de febrero y marzo de 1992; el segundo set de cortes se realizó con el corte cuatro en los meses de setiembre y octubre de 1992 y en noviembre-diciembre del mismo año el corte cinco. De las variables evaluadas solo la Fibra Neutro Detergente (FND), Materia Seca (MS) y el Forraje Seco por hectárea (FSH) resultaron altamente significativas $(\mathrm{P}<0,01)$.
\end{abstract}

\begin{abstract}
Evaluation of the potencial productivity of the black sorghum to forage (Sorghum almum). Information on productivity and nutritional composition of forage black sorghum or Columbus grass (Sorghum almum) was obtained though five cuts, 63 days long each one. The first cut was in October - November 1991, the second in December 1991 January 1992, the third in February - March 1992. A second set of cut took place with the fourth cut in September October 1992 and the fifth in November - December 1992. The variables evaluated were Neutral Detergent Fibrous (FND), Dry Matter (MS), Dry Forage by Hectarea (FSH) and Crude Protein (PC), but only FSH, MS and FND tumed to be significantly important $(\mathrm{P}<0,01)$.
\end{abstract}

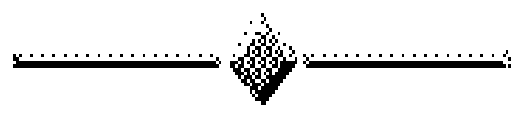

\section{INTRODUCCIÓN}

El sorgo negro follajero (Sorghm almum) adquiere relevancia en la actual necesidad de producción regional de semillas forrajeras de especies perennes y rústicas a bajo costo, y la posibilidad de obtener material forrajero de esta especie de condición perenne, aún en época de sequía acentuada. Esta gramínea de corte, que tiene alto rendimiento por unidad de área, gran capacidad de recuperación en la sequía, habilidad para soportar suelos encharcados, y de capacidad superior a la del maíz (Zea mays) y a los del género Pennisetum (Pennisetum purpureum) (Seifferty y Prates, 1978). Del sorgo negro forrajero se puede obtener más de una cosecha en su vida útil, lo que le da ventaja sobre otras especies usadas para este fin (Rodríguez, 1986).

Los ecotipos de sorgo negro forrajero (Sorghum almum) presentan considerables diferencias en lo concerniente a la duración del ciclo vegetativo, rendimiento, resistencia a plagas, resistencia a enfermedades, sensibilidad ante el estrés de humedad y utilización (Mela Mela, 1977; citado por Vega y Esperance, 1984).

La temperatura óptima para el desarrollo del sorgo es de 21 a $30^{\circ} \mathrm{C}$ y la mínima de $13^{\circ} \mathrm{C}$, temperaturas inferiores cesan el crecimiento (Escobar y Ramírez, 1970 citados por Vega y Esperance, 1984).

La precipitación media para una buena adaptación es de $500 \mathrm{~mm}$ (Hughes, 1974); no obstante los sorgos pueden crecer favorablemente en áreas con precipitaciones anuales de 750 mm o más (Whyte et al, 1964; citados por Vega y Esperance, 1984). Esta adaptación a la sequía, superior a la de otros cultivos, es debido a que las raíces de estas se desarrollan en forma lateral y son de profusa ramificación y amplia distribución (Robles, 1981), por las características de su sistema radicular, se hace posible que la planta pueda permanecer latente durante largos periodos de sequía. Una de sus limitantes

\footnotetext{
1 Presentado en la XLII Reunión Anual del PCCMCA en El Salvador, 1996. Proyecto de investigación financiado por la Universidad de Costa Rica.

2 Estación Experimental de Ganado Lechero Alfredo Vollo Mata. Facultad de Agronomía, Universidad de Costa Rica.

3 Escuela de Zootecnia. Facultad de Agronomía, Universidad de Costa Rica.
} 
es que en altitudes superiores a $1.900 \mathrm{msnm}$ el desarrollo es lento y se disminuye el porcentaje de germinación (Pitner, 1965; citado por Carrera, 1965).

Varios autores han encontrado una adecuada respuesta del sorgo a la fertilización nitrogenada, Alfaro (1988); Corrales (1986); Hernández (1986); Rivera (1967); Wall y Ross (1975); Zambrano y Torres (1974), con aumentos en su rendimiento y mejor su valor nutritivo.

El auge del cultivo del sorgo en América Latina, se ha debido en gran parte al interés de producir forr e como alimento de mantenimiento para rumiantes en época seca. Consecuentemente este interés a puesto en mayor disposición tierras que estaban ociosas, o que antes tenían otros usos, para que éstas fueran utilizadas en la siembra del sorgo (Martínez y Paul, 1993).

Oramas et al. (1993) define que la situación actual en Cuba, requiere la búsqueda de fuentes industriales de materia prima como alternativa a la supresión de importaciones de granos y semillas, inclusive de sorgo. Además agrega que el cultivo de sorgo ofrece perspectivas favorables en relación a otros granos, debido a que necesita menos requerimentos agrotécnicos en general y presenta una mayor plasticidad con respecto a la época de siembra y suelo.

Teniendo en cuenta las consideraciones anteriores, el presente trabajo pretende generar información sobre la productividad y valor nutricional del sorgo negro.

\section{MATERIALES Y MÉTODOS}

\section{Localización del estudio}

El experimento se realizó en la Estación Experimental de Ganado Lechero. Ing. Alfredo Volio Mata. de la Universidad de Costa Rica, situada en el Alto de Ochomogo, Distrito de San Rafael, cantón de la Unión, Provincia de Cartago, con una elevación de 1.545 msnm y una temperatura promedio de $15,5^{\circ} \mathrm{C}$ y un suelo que corresponde a un Typic Dystrandept. La variedad utilizada durante el ensayo fue sorgo negro forrajero (Sorghum almum). Se utilizó una parcela con un año de establecida y se evaluó la misma, con una distancia entre surcos de 0,70 y una fertilización considerada económica de $50 \mathrm{~kg} \mathrm{~N} / \mathrm{ha} /$ corte. El control de malezas se realizó después de cada corte, con glifosato (i.a). El estudio de campo se inició en julio de 1991 hasta marzo 1993.

En la Figura 1 se presentan los datos de precipitación correspondientes al periodo de estudio.

\section{Diseño Experimental}

Se utilizó un diseño experimental de bloques completos al azar con cinco cortes, nueve parcelas por corte, y cada parcela con tres repeticiones, con arreglo factorial $5 \times 9 \times 3$.

Cuadro 1. Datos mensuales de precipitación y temperatura de la Estación Experimental de Ganado Lechero. Ing. Alfredo Volio Mata. Cartago, Costa Rica.

\begin{tabular}{|c|c|c|c|c|c|c|}
\hline \multirow[t]{2}{*}{ Mes } & \multicolumn{2}{|c|}{ Año 91} & \multicolumn{2}{|c|}{ Año 92} & \multicolumn{2}{|c|}{ Año 93} \\
\hline & $\begin{array}{c}\text { P.(total) } \\
\text { (mm) }\end{array}$ & $\begin{array}{c}\text { T.(media) } \\
\left({ }^{\circ} \mathrm{C}\right)\end{array}$ & $\begin{array}{c}\text { P.(total) } \\
(\mathbf{m m})\end{array}$ & $\begin{array}{c}\text { T.(media) } \\
\left({ }^{\circ} \mathbf{C}\right)\end{array}$ & $\begin{array}{c}\text { P.(total) } \\
(\mathbf{m m})\end{array}$ & $\begin{array}{c}\text { T.(media) } \\
\left({ }^{\circ} \mathrm{C}\right)\end{array}$ \\
\hline Ene & 7,5 & 15,5 & 5,4 & 15,6 & 48,0 & 15,4 \\
\hline Feb & 18,1 & 15,3 & 51,6 & 16,0 & 9,9 & 15,4 \\
\hline Mar & 10,0 & 16,5 & 14,4 & 16,6 & 38,0 & 16,1 \\
\hline Abr & 46,1 & 16,9 & 40,4 & 17,0 & 63,5 & 17,1 \\
\hline May & 298,6 & 16,8 & 144,4 & 17,2 & 299,7 & 17,2 \\
\hline Jun & 184,0 & 17,2 & 246,6 & 17,1 & 411,3 & 17,1 \\
\hline Jul & 82,6 & 16,6 & 193,4 & 16,5 & 146,2 & 17,2 \\
\hline Ago & 160,6 & 16,8 & 218,4 & 16,6 & 147,2 & 17,2 \\
\hline Set & 217,4 & 17,1 & 372,0 & 16,1 & 360,0 & 16,2 \\
\hline Oct & 232,1 & 16,4 & 199,8 & 16,2 & 118,9 & 17,0 \\
\hline Nov & 103,2 & 16,4 & 114,8 & 16,2 & 88,8 & 15,9 \\
\hline Dic & 16,3 & 15,7 & 48,8 & 15,8 & 65,8 & 15,1 \\
\hline
\end{tabular}

Datos obtenidos en el Instituto Metereológico Nacional de Costa Rica.

P. $($ total $)=$ Precipitación total.

$\mathrm{T} .($ media $)=$ Temperatura media . 


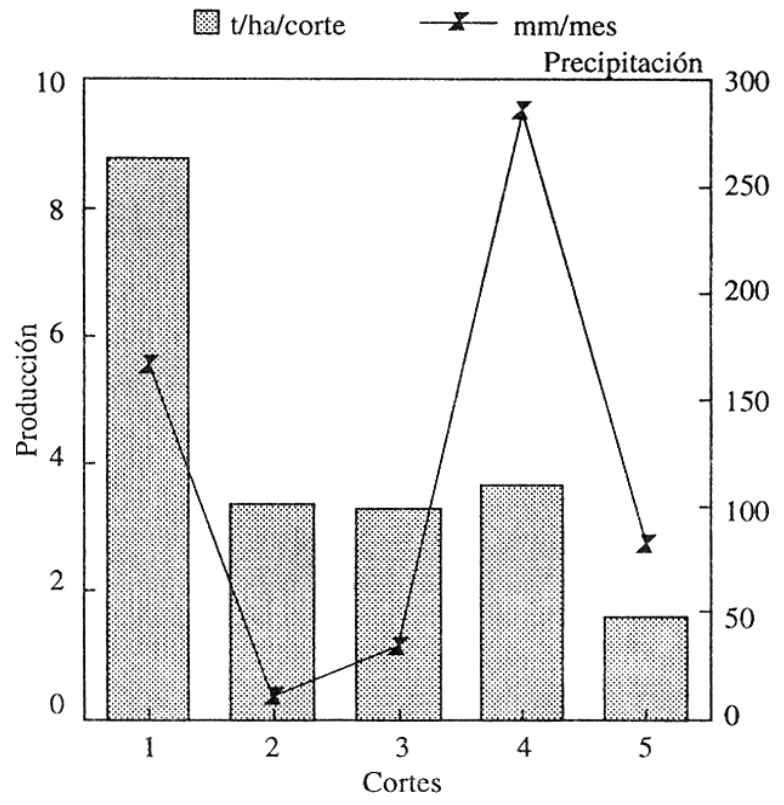

Fig. 1. Efecto de la época de corte sobre la variable forraje seco por hectárea en sorgo negro forrajero. Cartago, Costa Rica.

\section{Muestreo y Análisis de Calidad}

Se tomaron tres muestras de un metro lineal cada una cortadas a cinco $\mathrm{cm}$ de altura del suelo y se colectó todo el material aéreo, éste se cosechó una vez por semana, se pesó cada muestra en forma individual, luego se picaron las muestras y se homogenizaron para obtener una sola muestra de la cual se le realizaron los análisis bromatológicos correspondientes.

El porcentaje de Fibra neutro detergente (\% FND), ésta se analizó por medio de regresión cúbica, con un $\mathrm{r}^{2}$ $=0,40$.

\section{Análisis Estadístico}

Se realizó curvas de regresión para las diferentes variables evaluadas, para observar el comportamiento a través del tiempo, observándose para ello significancia $(\mathrm{P}<0,01)$ en las variables FSH, MS y FND en los cinco cortes analizados.

\section{RESULTADOS}

Al inicio de la investigación las parcelas fueron fertilizadas con $75 \mathrm{~kg}$ de $\mathrm{P}_{2} \mathrm{O}_{5} / \mathrm{ha}$. $\mathrm{Al}$ inicio del periodo de lluvias y después de esa aplicación con $50 \mathrm{~kg}$ de $\mathrm{N} / \mathrm{ha} /$ corte, en periodo seco no se efectuó riego y por lo tanto no hubo fertilización.

En el Cuadro 2, se compara investigación obtenida en esta investigación sobre el sorgo negro forrajero (Sorghum almum) y su potencial nutritivo, con diferentes fuentes forrajeras utilizadas en nuestro país (MAGIICA, 1991).

La variable FSH se analizó sobre regresión cúbica, con un $\mathrm{r}^{2}=0,58$ (Cuadro 3 ), el cual fue significativo, como es conocido el efecto de la precipitación es el que afecta más la expresión de los forrajes, por lo tanto se consideró necesario representar dentro de los gráficos el comportamiento de ésta a través de la investigación; como se puede observar en la Figura 1 el primer corte presentó una producción de $8.760 \mathrm{~kg}$ de $\mathrm{FSH} /$ ha/corte, mostrando la mejor producción de los cinco cortes, en el corte siguiente al disminuir la precipitación la producción llegó a $3.360 \mathrm{~kg} \mathrm{FSH} / \mathrm{ha} /$ corte, en el corte tres no mejoró la producción de sorgo ya que coincidió con la época más crítica de sequía y fue de $3.270 \mathrm{~kg}$ FSH/ha/corte. Después de este periodo se realizó un segundo set de muestreos, iniciando el mismo año pero en los meses de setiembre y octubre, con una producción promedio de $3.630 \mathrm{~kg} \mathrm{FSH} /$ ha/corte, y luego el último corte donde se obtuvo el rendimiento más bajo de 1.560 $\mathrm{kg} \mathrm{FSH/ha/corte.}$

Según Corrales (1986), trabajando con sorgo negro forrajero (Sorghum almum) con la variable FSH se obtuvo valores desde $5.136,95 \mathrm{~kg} / \mathrm{ha} /$ corte con el nivel 0 de nitrógeno y un valor de $7.738,34 \mathrm{~kg} / \mathrm{ha} /$ corte con el

Cuadro 2. Comparación de diferentes materiales forrajeros de corte. Cartago, Costa Rica, 1991-1993.

\begin{tabular}{lcccc}
\hline Material & $\begin{array}{c}\text { Edad } \\
\text { (días) }\end{array}$ & $\begin{array}{c}\text { Rendimiento } \\
\text { (t/MS/ha) }\end{array}$ & \% MS & \% PC \\
\hline Avena sativa & 70 & 2,85 & 17,14 & 17,38 \\
Triticale & 70 & 2,79 & 20,03 & 13,66 \\
Pennisetum sp & 70 & 9,28 & 17,07 & 12,09 \\
Sorghum almum* & 63 & 4,12 & 15,67 & 11,61 \\
\hline
\end{tabular}


Cuadro 3. Función de respuesta para las diferentes variables (FSH, \% MS, \% FND) de Sorgo Negro forrajero (Sorghum almum) en Ochomogo de Cartago, Costa Rica.

\begin{tabular}{lll}
\hline \multicolumn{1}{c}{ Parámetros } & Ecuación de regresión & \\
\hline FSH & $\mathrm{Y}=23,21-20,40 \mathrm{X}+6,58 \mathrm{X}(2)-0,67 \mathrm{X}(3)$ & $\mathrm{r}^{2}=0,58$ \\
$\%$ MS & $\mathrm{Y}=4,69+13,99 \mathrm{X}-5,52 \mathrm{X}(2)+0,66 \mathrm{X}(3)$ & $\mathrm{r}^{2}=0,40$ \\
$\%$ FND & $\mathrm{Y}=137,5-123,12 \mathrm{X}+74,62 \mathrm{X}(2)-18,18 \mathrm{X}(3)+1,53 \mathrm{X}(4)$ & $\mathrm{r}^{2}=0,40$ \\
\hline
\end{tabular}

nivel de $75 \mathrm{~kg} / \mathrm{N} / \mathrm{ha}$. Alfaro, 1988 indica valores de $5.324,2 \mathrm{~kg} / \mathrm{ha} /$ corte similares a los obtenidos por Avila, 1984; Crespo et al, 1981; Chao, 1982; Lima, 1980; Losilla, 1976; Ministerio de Agricultura del Perú, 1974 y Zelaya y Barea, 1969.

Con respecto al porcentaje de Materia Seca (\% MS), ésta se analizó por medio de regresión cúbica con un $\mathrm{r}^{2}=$ 0,40 con esta variable se observó un comportamiento normal donde el mayor porcentaje de MS se obtuvo en el corte cinco, y el menor valor se observó en el corte cuatro.

El porcentaje de MS estuvo inversamente relacionado con el contenido de humedad de la planta. Al reducirse la humedad del suelo por el efecto de la evaporación, hubo disminución en el contenido de humedad de la planta (transpiración) y por lo tanto un aumento significativo $(\mathrm{P}<0,01)$ en el porcentaje de MS.

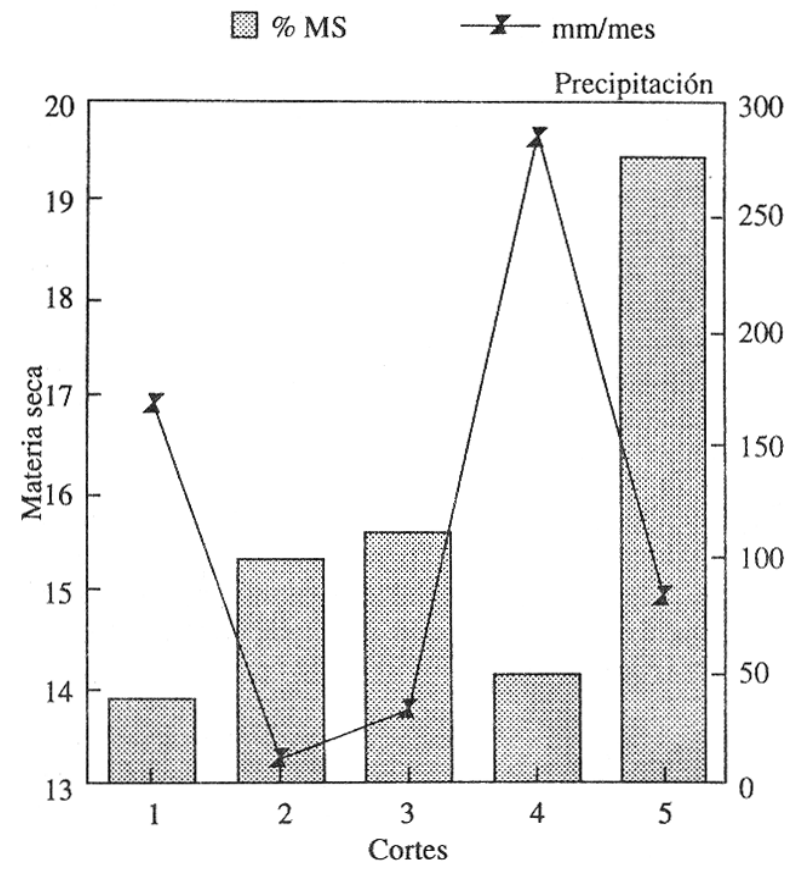

Fig. 2. Efecto de la época de corte sobre la variable porcentaje de materia seca en sorgo negro forrajero. Cartago, Costa Rica.
Los diferentes niveles de fertilización nitrogenada afectaron el porcentaje de MS, ya que conforme aumentó la fertilización nitrogenada, la planta presentó mayor tiempo para llegar asu madurez, y extendió su desarrollo vegetativo produciendo mayor biomasa, teniendo en cuenta que la precipitación es el factor determinante para una adsorción de nutrimentos. Los mayores porcentajes de MS se obtuvieron en el corte cinco con una precipitación promedio mensual de $82 \mathrm{~mm} / \mathrm{mes}$.

Según Pezo (1981), la determinación de la fibra neutro detergente, hace su fraccionamiento en el contenido celular y pared celular, obteniéndose como resultado los valores de los materiales digestibles totales (Contenido celular), los resultados obtenidos del análisis son variables, ya que son función del grado de lignificación del material.

En la Figura 3 se observa que en el corte uno y tres se obtuvo el mayor valor de ese componente con un 72,34 porciento y 72,76 respectivamente. Se observa que con respecto a los dos cortes anteriores no existió una relación con respecto a la cantidad de agua pluviométrica reportada; ya que con el corte cuatro se esperaría mayores valores de porcentaje de FND por una alta precipitación, pero no sucedió de esa forma. Esto se puede deber a un efecto de agotamiento de la planta por exposición a diferentes condiciones de estrés a través de las evaluaciones.

Otro efecto es el comportamiento de la fertilidad sobre el porcentaje de FND, el cual al aumentar el nivel de fertilizante nitrogenado se observó una tendencia a disminuir la proporción de este componente. Muldoon y Pearson (1979), determinaron que el porcentaje de digestibilidad de la materia orgánica disminuyó conforme aumentó la edad del forraje y esto está relacionado con el porcentaje de FND debido a una disminución en el contenido de carbohidratos solubles y aumento en los carbohidratos estructurales.

Los valores obtenidos sobre porcentaje de FND nos orientan a determinar un material de alta digestibilidad con un promedio de $70,48 \%$ 


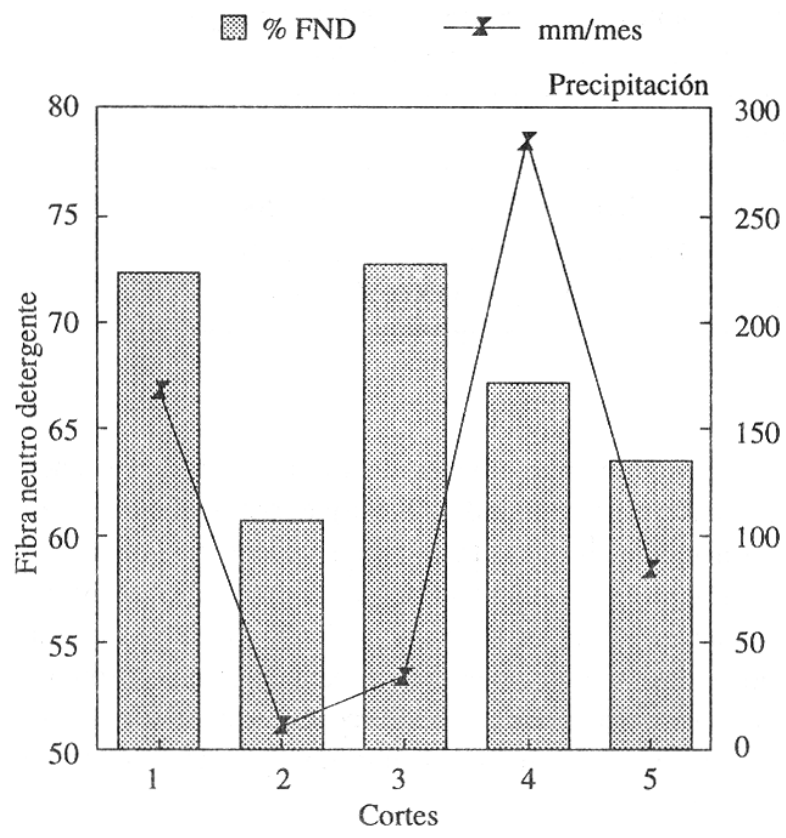

Fig. 3. Efecto de la época de corte sobre la variable porcentaje de fibra neutro detergente en sorgo negro forrajero. Cartago, Costa Rica.

La función de respuesta de las diferentes variables estudiadas del sorgo negro forrajero, se presentan en el Cuadro 3.

\section{LITERATURA CITADA}

ALFARO, G.O.L 1988. Evaluación de la producción y calidad del sorgo negro forrajero (Sorghum almum) a través de diferentes distancias de siembra, densidades de siembra y niveles de fertilización nitrogenada. Tesis Ing. Agron. Zoot. San José, Costa Rica. Universidad de Costa Rica. 108 p.

ARROYO, R; AGUIRRE, D; CAMACHO, 1; EDUARTE, E; VILLALOBOS, L. 1993. Evaluación del potencial productivo del pasto King Grass.(Pennisetum purpureum var King Grass) durante las épocas seca y lluviosa. I. Análisis de características de calidad. In resúmenes de trabajos científicos del IX Congreso Nacional Agronómico y de Recursos Naturales. San José, Costa Rica. 146 p.

ARROYO, R; AGUIRRE, D; CAMACHO, 1; EDUARTE, E; VILLALOBOS, L. 1993. Evaluación del potencial productivo del pasto King Grass (Pennisetum purpureum var King Grass) durante las épocas seca y lluviosa. II. Análisis de características de calidad. In resúmenes de trabajos científicos del IX Congreso Nacional Agronómico y de Recursos Naturales. San José, Costa Rica. $147 \mathrm{p}$.
AVILA, G. M. E. 1984. Efecto de la frecuencia de corte y la fertilización nitrogenada, sobre el rendimiento, composición química y digestibilidad "In vitro" de la materia seca del pasto King Grass (Pennisetum purpureum) en época seca. Tesis Ing. Agr. San José,Costa Rica. Universidad de Costa Rica. 56 p.

CARRERA, A. M. 1965. Estudio comparativo de rendimientos de sorgos forrajeros (Sorghum vulgare pers) en la zona del Pacífico norte. Tesis Ing. Agr. San José, Costa Rica. Universidad de Costa Rica. 45 p.

CHAO, L. 1982. The response of napier grass (Pennisetum purpureum) to animal manure and chemical fertilizer. Effects on dry matter, yield and quality. $\mathrm{J}$ oumal of the Agricultural Association of China. Serie 119: 64 - 67 p.

CORRALES, S. J. C. 1986. Efecto de la densidad y distancia de la siembra y la fertilización nitrogenada sobre la producción de biomasa y la calidad del sorgo negro forrajero (Sorghum almum). Tesis Ing. Agron. Zoot. San José, Costa Rica. Universidad de Costa Rica. 173 p.

CRESPO, G; RAMOS, N; SUAREZ, J; HERRERA, S; GONZALES, S. 1981. Producción y calidad de los pastos. Revista Cubana de Ciencias Agrícolas. Cuba. 15(2): 211-225 p.

HERNANDEZ, H. E. 1986. Fertilización nitrogenada y edad de corte para sorgo negro forrajero (Sorghum almum). Tesis Ing Agr. San José, Costa Rica. Universidad de Costa Rica. 145 p.

HUGHES, H. D. 1974. Forrajes. 4 imp. Editorial Continental. s. a. México. 758 p.

INSTITUTO COLOMBIANO AGROPECUARIO. PASTOS Y FORRAJES. Compendio $\mathrm{N}^{\circ}$ 30. Medellín, Colombia. $375 \mathrm{p}$.

LIMA, P. 1980. Efeito do método de semeadura e aduracáo nitrogenada sobre o rendirniento de sorgo forrajeiro (Sorghum sudonensex s. bicolor) e milheto (Pennisetum americanum schum) consorciados com feijáo rniudo (Vignia unguiculata (L) walp.) ou adubacados con nitrógenio. U.E.P.A. de Pelotas, Brasil. 89 - 91 p.

LOSILLA, B. 1976. Efecto de diferentes niveles de fertilización y frecuencia de corte en el rendimiento, composición química y digestibilidad "in vitro" del pasto gigante (Pennisetum purpureum). Tesis. Ing Agr. San José, Costa Rica. Universidad de Costa Rica. 67p.

MAG - IICA. 1991. Estudio a nivel nacional para mejorar la tegnología de la alimentación de ganado lechero a través del uso de forrajes de corte. Editado por G. Cubillos. San José. Costa Rica. 285 p.

MARTINEZ, O; PAUL, C. 1993. Evaluación de cuatro varie dades de sorgo (Sorghum bicolor L. Moench) de doble 
propósito (Grano y rastrojo). In XXXI Reunión Anual del Programa Cooperativo Centroamericano para el Mejoramiento de Cultivos y Animales (PCCMCA). Guatemala. 239 - 241p.

MULDOOM, D.K; PEARSON,J. 1979. Thehibridbetween Pennisetum americanum and Pennisetum purpureum. Herbage Abstracts 49(5): 189 - 199.

ORAMAS, G; TORRES, C; BETANCOUR, J; GARCÍA, E. 1993. Evaluación de nuevas variedades de sorgo (Sorghum bicolor L. Moench) de grano para Cuba. In XXXI Reunión Anual del Programa Cooperativo Centroamericano para el Mejoramiento de Cultivos y Animales (PCCMCA). Guatemala. 253 - 256 p.

PEZO. D. 1981. La calidad nutritiva de los forrajes. In compendio de producción y utilización de forrajes en el trópico, Materiales de enseñanza n. 10. CA TIE. Turrialba, Costa Rica. 129 p.

RIVERA, G. J. 1967. Efecto de la fertilización en la producción de materia verde en dos variedades de sorgo forrajero. Tesis Ing. Agr. Managua, Nicaragua. Escuela Nacional de Agricultura y Ganadería. 37 p.

ROBLES, S. R. 1981. Producción de granos y forrajes. segunda edición. Editoml Limusa. México D.F., México. $141-170 \mathrm{p}$
RODRIGUEZ, I. 1986. Cambios en la fermentación del sorgo negro forrajero (Sorghum almum) en microsilos con diferentes niveles de melaza y tamaños de partícula y tres edades de corte. Tesis Ing. Agron. Zoot. San José, Costa Rica. Universidad de Costa Rica. 82 p.

SEIFFER, N. F; PRATES, E. R. 1978. Forrajeiras para ensilagem; valor nutritivo e qualidade de ensilagem de cultivares de milho (Zea mays), sorgos (Sorghum sp) e milhetos (Pennisetum americanum, schum). Rev. Soc. Bras. Zoot. 7(2): 183 - 195.

VEGA, S; ESPERANCE, M. 1984. Sorgo forrajero (Sorghum bicolor). Pastos y forrajes. Cuba. 7(1). $1-15$ p.

WALL, S. J; ROSS, W. 1975. Producción y usos del sorgo. Buenos Aires, Argentina. Editorial Hemisferio Sur. 399p.

ZAMBRANO, R. R; TORRES, O. E. 1974. Cultivo del sorgo forrajero. Boletín técnico $N^{\circ}$ 79. Lima, Perú. Ministerio de Agricultura. $27 \mathrm{p}$.

ZELAYA, H; BAREA, F. 1969. Fertilización nitrogenada en sorgo forrajero (Sorghum vulgare pers) y su optimización económica. Tesis. Ing. Agr. Managua, Nicaragua. Escuela Nac. de Agricultura y Ganadería. 78p. 